\title{
Análisis de la relación entre alteraciones del eje hipotálamo-hipófisis-tiroides y exposición a perclorato en ratones de la cepa ICR
}

\author{
Germán Javier Arias Holguín Msc ${ }^{1}$, Miriam Andrea Wilches Torres Msc², \\ Diego Alejandro Pedraza ${ }^{2}$
}

${ }^{1}$ Docente de Tiempo completo Departamento de Química y Bioquímica, Director de Formación y Capacitación Docente Universidad de Boyacá. ${ }^{2}$ Docente Departamento de Química y Bioquímica Universidad de Boyacá

${ }^{3}$ Estudiante de Medicina Universidad de Boyacá.

Correspondencia: gjarias@uniboyaca.edu.co

Recibido: 15/03/2013 Aceptado: 24/05/2013

\begin{abstract}
Resumen
Este estudio establece una correlación entre la exposición a perclorato de amonio y la presencia clínica de alteraciones en el eje hipotálamo-hipófisis- tiroides, tomando como referencia diferentes dosis de aplicación desde la aceptada como dosis segura hasta un incremento significativo de dicha dosis. El trabajo reviste gran importancia debido a que esta sustancia química es uno de los compuestos de mayor uso como pesticida en el departamento de Boyacá. Mediante ensayos inmunoenzimáticos con microplacas; con los Kit comerciales Kit Accubind Elisa Microwells TSH y Kit Accubind Elisa Microwells T4L y el análisis clínico se pudo establecer la existencia de alteraciones en el eje hormonal, lo que puede ser indicador en el futuro de un alto riesgo por parte de los individuos que manipulen esta sustancia.
\end{abstract}

Palabras clave: Eje hipotálamo-hipófisis-tiroides, perclorato de amonio, hormona estimulante de toroides, tetrayodotirosina, hipertiroidismo, hipotiroidismo.

\section{Analysis of alterations between the hypothalamic-pituitary-thyroid axis and perchlorate exposure in ICR mice strain}

\begin{abstract}
This study establishes a correlation between exposure and clinical alterations in the hypothalamic-pituitary-thyroid axis. Different dosages were applied, from the accepted safe dosage up to a significant increase dosage. The study was carried out under the laboratory animal center conditions at Universidad of Boyacá (Colombia). Due that ammonium perchlorate is one of the most used compounds as pesticide in Boyacá department this type of studies are extremely important. Using enzyme immunoassay in micro-plate (Accubind Elisa Microwells TSH and Kit Accubind Elisa Microwells T4L ) and following clinical analysis alterations in the hormonal axis were found which could be be indicative of a possible high risk in the future for individuals who handle this substance.
\end{abstract}

Keywords: hypothalamic-pituitary-thyroid axis, ammonium perchlorate, thyroid stimulating hormone, tetraiodothyronine, hyperthyroidism, hypothyroidism. 


\section{Introducción}

Los percloratos por sus características físicoquímicas han sido diseminados a lo largo de la industria en los últimos años. Desde la década de los 50 se han descrito aproximaciones del mecanismo de acción de estas sustancias en el organismo, por lo que se empezaron a utilizar en el tratamiento de la tirotoxicosis.

Sin embargo, más adelante se presentaron varios casos de una letal enfermedad llamada anemia aplásica, hecho que obligó a retirar del mercado estas sustancias.

Se han encontrado altas concentraciones de percloratos en otras partes del mundo como es el caso de Chile, Israel y Estados Unidos. En los últimos años se ha esclarecido, en alguna medida, el mecanismo de acción de los percloratos y cómo pueden desarrollar trastornos asociados al metabolismo del yodo.

Los percloratos son estables a temperatura ambiente, pero al elevar su temperatura se convierten en moléculas altamente reactivas que generan calor y explosión. Debido a este comportamiento, los percloratos se utilizan como propulsores de cohetes, fuegos artificiales, señales luminosas, pólvora y explosivos.

A finales de la década de los 50 , un estudio donde se analizaron diferentes sustancias antitiroideas entre ellas; los percloratos, estableció un nexo epidemiológico con el hipotiroidismo por lo que se usó en pacientes hipertiroideos. A los pocos años los percloratos fueron retirados del mercado por los efectos adversos ya comentados, en 1993 se comprobó que los percloratos no solo actúan sobre el simportador sodio-yodo (NIS) sino que también actúan sobre las proteínas trasportadoras en sangre lo que genera mayor eliminación de T4 Libre y desestabilización del eje hipotálamo, hipófisis, tiroides (1).

Varios estudios en la última década evidenciaron niveles normales de T4 después de la exposición a percloratos en corto y largo plazo. Sin embargo, hay más reportes de estudios que informan claros cambios fisiológicos a concentraciones por debajo de las denominadas seguras (2).

Sin embargo, hay aún muchos nexos epidemiológicos desconocidos referentes a la relación existente entre hipotiroidismo y exposición a percloratos, así que el desarrollo de investigaciones permitirá obtener datos e información que conlleven a esclarecer la acción de los percloratos en la salud.

Por otra parte, el hipotiroidismo es una de las patologías endocrinológicas más prevalentes en la población mundial en la que se requiere un amplio apoyo interdisciplinario para su manejo. Se ha establecido que su incidencia aumenta con el paso de los ańos; actualmente se considera una prevalencia a nivel mundial cercana al $5 \%$ y en Colombia alrededor del $2 \%$ de la población general y del 5 al $10 \%$ en mujeres postmenopáusicas (3).

El mecanismo de acción de los percloratos sobre el metabolismo del yodo en el ser humano se ha establecido como una inhibición competitiva sobre un receptor proteico específico denominado NIS (simportador de yodo-sodio) generando disminución en la producción de hormonas tiroideas y aumento de la hormona estimulante de tiroides (TSH), conociéndose este fenómeno como hipotiroidismo (4).

Percloratos. El término perclorato o anión perclorato se refieren a un grupo de átomos con carga negativa que están formados por un átomo de cloro unido a cuatro átomos de oxígeno. La fórmula molecular del perclorato es $\mathrm{ClO}_{4}^{-}$.

Los términos percloratos o sales de perclorato se refieren a los compuestos inorgánicos que contienen el anión perclorato unido a un grupo con carga positiva como por ejemplo amonio o a un metal alcalino o alcalinotérreo, los percloratos son incoloros y no tienen olor. 
Hay cinco tipos de percloratos que se manufacturan en grandes cantidades: perclorato de magnesio, perclorato de potasio, perclorato de amonio, perclorato de sodio y perclorato de litio. Los percloratos se encuentran en dos formas en el ambiente, ya sea como sólidos o disueltos en agua.

Aproximadamente el $90 \%$ de los percloratos son fabricados por el hombre en forma de perclorato de amonio, el cual es usado como propulsor de cohetes y misiles. Las sales de perclorato son también usadas en bolsas de aire, reactores nucleares, plantas eléctricas, refinerías de aluminio, aceites lubricantes, producción de pinturas, pirotecnia, fabricación de munición y propulsores de cohetes espaciales (3-5).

Otros usos de los percloratos incluyen; pegamentos, baños electrolíticos, baterías, agentes para desecar y grabado, agentes para limpia y blanquear y sistemas para generar oxígeno. Poco se sabe acerca de la naturaleza, la cantidad y la posibilidad de liberación al ambiente desde estas posibles fuentes de perclorato. El perclorato también se usa para contrarrestar los efectos adversos de la droga amiodarona, usada para tratar arritmias cardíacas $y$ angina (6).

Propiedades de los percloratos. Las sales de perclorato son altamente solubles en agua, su movilidad es importante en fuentes y superficies acuosas. Bajo aguas subterráneas y en condiciones habituales, en la superficie del agua, es considerado como un elemento químicamente inerte. Por estas condiciones la contaminación del agua permanece por largos períodos de tiempo. Algunos estudios mencionan como principal fuente de exposición a percloratos las comidas aún más que en el consumo de agua.

Teniendo en cuenta la localización del simportador de sodio-yodo en el cuerpo se iniciaron estudios de su presencia en la glándula mamaria y evidenciaron su excreción por leche materna aumentando la relevancia del problema sobre todo para infantes lactantes (3).
El ión perclorato tiene una forma iónica con carga similar al yodo y puede competir con la recaptación de yodo por el simportador tiroideo sodio-yodo, presente en la superficie de las células y encargado de trasportar el yodo del plasma al interior de la tiroides. Este simportador tiene 30 veces más selectividad por los percloratos que por el yodo $(3,4)$.

La agencia de protección ambiental de los Estados Unidos, presentó una dosis de referencia para percloratos de $0.7 \mathrm{ug} / \mathrm{Kg} / \mathrm{dí}$, permitiendo establecerse esta dosis como segura de efectos adversos en humanos.

Epidemiología de los efectos adversos por exposición a los percloratos. Diversos estudios han evaluado la evidencia de los efectos adversos de los percloratos sobre la salud en el ser humano. Un estudio realizado en USA ha registrado niveles de los tres inhibidores del NIS en muestras recogidas en orina por NHANES durante los años 2001-2002.

Una Investigación en Israel reportó concentraciones séricas de los tres inhibidores de NIS en poblaciones con diferentes niveles de perclorato en la superficie de agua potable de su comunidad (7).

Se han realizado otros estudios donde al parecer no hay un vínculo estadísticamente significativo entre el aumento de TSH y disminución de T4 con la exposición a percloratos. Así mismo, el aparente sinergismo entre dos sustancias bociógenas como son los tiocinatos y los percloaratos en mujeres con aumento de los niveles de TSH son al parecer dependientes de los niveles de yodo (8).

Por otra parte, los trabajadores de las plantas de producción de perclorato de amonio se encuentran expuestos a concentraciones mayores que cualquier otra persona de la población general. Una investigación realizada en unos trabajadores productores de perclorato de amonio por 27 años, donde se evaluó T4 total, T3 total, TSH y T4L no encontró diferencias entre los trabajadores expuestos y los no expuestos (9). 
Estudios recientes han encontrado contaminación significativa de percloratos en aguas subterráneas en el oeste de USA como resultado de la eliminación de perclorato de amonio y lixiviación de la industria $(4,10)$.

Un estudio en el NHANES de 2820 residentes de Estados Unidos en el 2001-2002 reporta que todos los residentes presentaban niveles de percloratos en orina.

Trabajadores expuestos a perclorato por vía inhalatoria a concentraciones de hasta $34 \mathrm{mg} /$ día en un promedio de tres años no evidenció cambios en la estructura ni en la concentración sérica de hormonas tiroideas. Sin embargo, después de tres turnos seguidos de 12 horas de exposición se observó una inhibición de la recaptación de yodo de hasta un $38 \%$.

Yodo. En la vida de todos los mamíferos el yodo es un elemento esencial. Su única función fisiológica conocida implica su presencia en las hormonas tiroxina y triyodotironina (3).

El yodo es ingerido por vía oral y es absorbido en el tracto gastrointestinal como un anión yoduro. El yodo como el yoduro es transportado desde el flujo sanguíneo al interior de la glándula tiroides por el NIS donde son sintetizadas las hormonas T4 y T3. De retorno al flujo sanguíneo la hormona $\mathrm{T} 4$ es desiodinada a T3 su forma activa.

Cuando las concentraciones de T4 y T3 están bajas, en el torrente sanguíneo, el hipotálamo sintetiza la hormona liberadora de tiroides (TRH). Esta estimula la glándula pituitaria para producir la hormona estimulante de tiroides (TSH); hay un receptor para TSH en la glándula tiroides donde al unirse da origen al estímulo productor de T4, esta induce la producción de tiroglobulina, peroxidasa tiroidea, NIS y tiroxina (4). Este sistema de retroalimentación negativa intenta mantener constantes los niveles de hormonas tiroideas en sangre $(3,5)$.
La recaptación de yodo en la glándula tiroides está facilitada por un mecanismo coordinado por el NIS el cual permite acumular en el tejido tiroideo yodo a concentraciones $20-40$ veces mayores que las encontradas en el plasma. El simportador proteico de sodio/yodo permite recaptar yodo con sodio pero posteriormente libera el sodio e ingresa el yodo. El NIS es sensible al estímulo tanto del yodo como de la TSH, sin la acción de ésta el yodo no debería ser importado (4).

\section{Requerimientos diarios}

Los requerimientos diarios de yodo para humanos son los siguientes, Tabla 1 (11):

Tabla 1. Requerimientos diarios de yodo para humanos

\begin{tabular}{ll} 
Infantes (1-12 meses) & $50 \mu \mathrm{g}$ \\
\hline Niños (2-6 años) & $90 \mu \mathrm{g}$ \\
Escolares (7-12 años) & $120 \mu \mathrm{mg}$ \\
\hline Adultos (mayor de 12 años) & $150 \mu \mathrm{g}$ \\
Mujeres gestantes y lactantes & $200 \mu \mathrm{g}$ \\
\hline
\end{tabular}

Un adulto normal gasta 80ug de yoduro al día en la producción de hormonas tiroideas. De la ingesta diaria de yodo solo un 35\% se queda en la glándula recirculando, compensando la pérdida de éste por heces y orina producto del metabolismo (3).

La mayor reserva de yodo se encuentra en los océanos. Sin embargo, está presente en concentraciones relativamente bajas $(60 \mathrm{ug} / \mathrm{L})$. El principal mecanismo de acción de los percloratos sobre la glándula tiroides es la inhibición del simportador proteico sodio/yodo (NIS), el NIS es responsable de la translocación activa de ambos (yodo y perclorato) desde el torrente sanguíneo al folículo tiroideo (1).

El perclorato de amonio ha sido empleado para determinar la función tiroidea al ser administrado para evaluar la recaptación tiroidea de yodo marcado, se observó la salida de yodo de la glándula tiroides al torrente sanguíneo y se cree que es por 
difusión ya que el NIS mantiene la diferencia del gradiente de concentración (1).

Un estudio realizado en 1959 desarrolló una comparación de diferentes agentes antitiroideos incluyendo tiocianatos, yoduro de potasio y perclorato, en el que se concluyó que el perclorato no altera significativamente la síntesis de monoyodotirosina (MIT) y diyodotirosina (DIT) (1). Curiosamente, en el año 1966 otros investigadores ponen en tela de juicio el mecanismo de acción de estos compuestos ya que comprobaron una acción directa de estos sobre el MIT y el DIT.

En el año de 1993 Okabe reporta que el perclorato desplaza la T4 que está unida a la albúmina. Esto implica que la T4L desplazada se elimina más rápidamente dando lugar a una alteración en el eje del hipotálamo (1). La tasa de recaptación diaria de percloratos oscila entre $10-1000 \mathrm{mg} / \mathrm{Kg}$.

Finalmente, en el año 2008 se encuentra evidencia de que el perclorato de amonio es transportado al interior de la glándula gracias a un estudio donde analizan el comportamiento de TSH con la exposición a percloratos en dosis específicas (1).

\section{Desórdenes relacionados con el yodo.}

Una dosis alta de yodo puede generar efectos variables. Sin embargo, todo depende de la salud de la glándula, en casos como estos el balance homeostático es regulado por un mecanismo llamado efecto de Wolff-Chaikoff. La glándula tiroides no produce más hormonas tiroideas hasta que se adapte a las altas concentraciones de yodo intracelular, la glándula tiroides se libera del efecto de Wolff-Chaikoff cuando la concentración de hormonas desciende lo suficiente como para estimular el NIS y reiniciar el proceso. Por lo general este efecto solo continúa por unos días bajo una continua y excesiva dosis de yodo (3).

Ciertos desórdenes como la enfermedad de Graves y Hashimoto pueden generar en algunos sujetos tiroiditis, aunque estas patologías tampoco se es- capan del efecto de Wolff-Chaikoff, la tiroides no está funcionando óptimamente y ésta no es capaz de reanudar la producción de hormonas tiroideas, desencadenando hipotiroidismo.

Hay otros desórdenes tiroideos donde la glándula produce hormonas independientemente de las concentraciones séricas, esta condición es conocida como tirotoxicosis de Job-Basedow; ocurre cuando los nódulos tiroideos tienen una función independiente al control de la TRH y TSH. Cuando esto ocurre la producción de hormonas tiroideas es directamente proporcional a la ingesta de yodo (3).

La deficiencia de yodo puede causar una amplia variedad de efectos adversos por la inadecuada concentración de hormonas tiroideas; este grupo de trastornos se le conoce bajo el título de desórdenes por deficiencia de yodo (3).

El objetivo del yodo como suplemento dietario es mantener menos del $50 \%$ de la población con concentraciones de yodo en orina en rangos menores a $100 \mathrm{ug} / \mathrm{L}$ y menos del $20 \%$ de la población con concentraciones menores de $50 \mathrm{ug} / \mathrm{L}$.

Un yodo urinario deseado de $100 \mathrm{ug} / \mathrm{L}$ fue establecido porque esta concentración representa una recaptación de yodo aproximada de $150 \mathrm{ug} / \mathrm{L}$.

El hipotiroidismo fue definido clínicamente significativo con; TSH $>4.5 \mathrm{mU} / \mathrm{L}$ y tiroxina $(\mathrm{T} 4)<4.5$ ug/dL, subclínico o leve con una TSH $>4.5 \mathrm{mU} / \mathrm{L}$ y T4> $4.5 \mathrm{ug} / \mathrm{dL}$. El hipertiroidismo fue definido con TSH <0.1 mU/L y T4 > 13.2 ug/dl; subclínico o leve con TSH $<0.1 \mathrm{mU} / \mathrm{L}$ y T4 $<13.2 \mathrm{ug} / \mathrm{dL}$. Los valores normales de TSH; 0.3-5.01 mU/L y valores normales de T4 5-12 ug/dL (4).

Por su parte evidencias de The National Health and Nutrition Examination Survey (NHANES) 1999-2002 mostraron una prevalencia de hipotiroidismo por TSH $>4.5 \mathrm{mU} / \mathrm{L}$ aproximada de $3.7 \%$, el hipotiroidismo en mujeres de edad reproductiva fue de $3.1 \%$ (12). 
La deficiencia de yodo de carácter prolongado puede desencadenar hipotiroidismo. En adultos algunos síntomas tempranos son debilidad, fatiga, intolerancia al frío, constipación, ganancia de peso, depresión, fragilidad en uńas, dolor articular o muscular, cabello fino y quebradizo y palidez. Rara vez los síntomas empeoran por deficiencia de yodo.

Por otro lado la deficiencia de yodo en fetos, infantes o niños tiende a ser más serio ya que este influye en el desarrollo neuronal, si ésta se presenta durante la etapa fetal se han observado abortos espontáneos o nacimientos con producto muerto.

\section{Metodología}

Este trabajo constituye una investigación analítica de diseńo experimental con grupo control, donde se tomaron muestras de sangre de ratones de la cepa ICR expuestos a perclorato de amonio durante 14 días. La metodología utilizada fue la siguiente:

\section{Adaptación del modelo animal a las condiciones} del bioterio de la Universidad de Boyacá. Los ratones fueron mantenidos en condiciones de luz, temperatura (entre $20^{\circ} \mathrm{C}$ y $22^{\circ} \mathrm{C}$ ) y ruido ideales para reducir al mínimo el estrés. Estos parámetros fueron controlados en el bioterio, gracias al equipo Enviro-GardTM-B 59016N. Cada grupo de ratones fue introducido en un habitáculo previamente esterilizado.

2. Exposición a perclorato de amonio: Los ratones fueron expuestos a perclorato de amonio disuelto en agua potable, el cual se suministró vía oral a cada individuo según el grupo correspondiente:

Grupo 1: Grupo Control. Ratones sin exposición a perclorato.

Grupo 2: Grupo Dosis Segura. Ratones expuestos a dosis segura de perclorato, según OMS. (0.7ug/Kg/día)
Grupo 3: Grupo Dosis Duplicada. Ratones expuestos al doble de la dosis segura de perclorato.

Grupo 4: Grupo Dosis Quintuplicada. Ratones expuestos a cinco veces la dosis segura de perclorato.

Grupo 5: Grupo Dosis Decaplicada. Ratones expuestos a diez veces la dosis segura de perclorato.

Cada grupo experimental estuvo constituido por cinco ratones hembra adultas de la cepa ICR.

3. Sangria y toma de muestra: La muestra que se analizó fue el suero obtenido a partir de la sangría total de cada individuo. Las muestras se colectaron en tubos estériles para tal fin, y se analizó la concentración de hormona TSH utilizando pruebas rápidas de detección.

4. Procesamiento y análisis de muestras: Se realizaron mediciones de la concentración de hormonas TSH y T4 Libre, utilizando pruebas rápidas de detección. Los cuales se hicieron mediante ensayo inmunoenzimático con microplacas con los Kit comerciales Kit Accubind Elisa Microwells TSH y Kit Accubind Elisa Microwells T4L.

El tratamiento de los ratones se realizó teniendo en cuenta la resolución No 008430 de 1993, artículo 87 del Ministerio de Salud República de Colombia; que indica que los animales seleccionados para la experimentación deben ser de una especie y calidad apropiada, y utilizar el mínimo número requerido para obtener resultados científicamente válidos. De igual forma todos los materiales y reactivos utilizados en el laboratorio de investigación fueron dispuestos en recipientes de recolección de residuos químicos, de acuerdo con las normas establecidas.

\section{Niveles hormonales}

\section{Resultados}

Una vez obtenido el suero, a partir de sangre obtenida de las ratonas previamente expuestas a 
perclorato de amonio, se realizó la medición de la concentración de las hormonas TSH y T4L, teniendo en cuenta los niveles normales:

$$
\begin{gathered}
\mathrm{TSH}=0.39-6.16 u \mathrm{UI} / \mathrm{mL} \\
\mathrm{T} 4 \mathrm{~L}=0.8-2.00 \mathrm{ng} / \mathrm{dL} .
\end{gathered}
$$

Los datos reportados para cada grupo fueron producto del promedio de la concentración de las hormonas medidas en cada animal de experimentación, teniendo en cuenta la similitud de cada uno de los valores tomados en cada animal y con el fin de tener un reporte mucho más acertado, se tomó el promedio de la concentración.

El grupo Dosis Segura presentó una TSH disminuida y una T4L normal, debido según las revisiones realizadas a mecanismos alternos que mantienen la producción de T4L a pesar del nivel disminuido de TSH.

Los grupos expuestos a dosis mayores a la segura presentaron niveles de TSH dentro del rango normal, mientras que los niveles de T4 estuvieron incrementados, sugiriendo que los mecanismos alternos reguladores de la producción hormonal pueden afectarse a medida que hay mayor exposición a perclorato de amonio.

En la figura 1 se observa que el grupo Control Negativo, presentó niveles normales de las hormonas TSH y T4L.

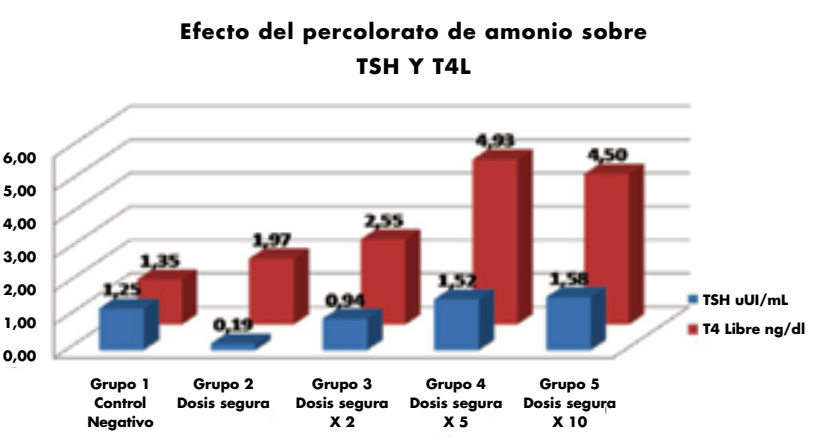

Figura 1. Concentración de las hormonas TSH y T4L en sangre de ratones expuestos a perclorato.
El grupo denominado Control Negativo presentó un nivel para TSH de $1,25 \mathrm{uUI} / \mathrm{mL}$ y de T4L de ng/dL lo que permitió establecer un nivel normal de hormonas y se convirtió en un punto de análisis para los resultados obtenidos en ratones expuestos a perclorato de amonio.

Para el grupo denominado Dosis Segura (0.7ug/ $\mathrm{Kg} / \mathrm{d}$ ía) los niveles encontrados fueron: TSH de $0,19 \mathrm{uUI} / \mathrm{mL}$ y T4L DE $1,97 \mathrm{ng} / \mathrm{dL}$, valores que demuestran que posiblemente el perclorato de amonio ha generado algún efecto sobre la TSH, ya que el valor se presenta de forma disminuida aunque T4L se mantiene dentro del rango normal pero acercándose al límite superior, lo que pone de presente que la sustancia utilizada genera cambio a nivel endocrino dentro de los animales utilizados.

El grupo Dosis Segura x2, presentó los siguientes niveles hormonales; TSH 0,94uUI/mL y T4L de $2,55 \mathrm{ng} / \mathrm{dL}$. A partir de este grupo los valores séricos de las hormonas toman un comportamiento uniforme, es decir para TSH se mantienen dentro del rango normal pero T4L sale del rango normal presentando un aumento notorio. Este hecho evidencia que a medida que se aumenta la dosis de perclorato; TSH se mantiene mientras que T4L aumenta considerablemente.

En el grupo Dosis Segura $x 5$ se presentaron niveles de TSH 1,52 uUI/mL y T4L de 4,93 ng/dL. Por su parte, el grupo Dosis Segura x10 manifiesta mucho más la tendencia hormonal con valores de TSH 1,58 uUI/mL y T4L 4,50ng/dL.

Comportamiento. El permanente seguimiento comportamental de los ratones mostró; hiperactividad en los grupos expuestos a dosis mayores a la segura, al ser comparados con el grupo control. Sugiriendo un posible hipertiroidismo en estos grupos que parece estar relacionada con la afectación directa al eje hipotálamo - hipofisario. 


\section{Discusión}

El efecto nocivo del perclorato de amonio sobre la producción de hormonas tiroideas no se relaciona únicamente con la disminución hormonal, como reportan la mayoría de los estudios, sino que también puede generar una sobreproducción de hormonas tiroideas $(11,12)$.

En el Grupo Control se observó; en cuanto al peso, que fueron ganando peso progresivamente debido al proceso de crianza dentro del bioterio. Así mismo, los valores hormonales se convirtieron en la base para poder hacer el análisis porque estuvieron dentro de los rangos normales, lo que supone que no hubo ningún efecto que alterara el proceso de crianza.

El Grupo Dosis Segura, en cuanto al peso; fue ganado progresivamente de manera normal. Sin embargo, con respecto al grupo control, luego de la mitad del tratamiento y al final del mismo el peso ganado fue menor. Aspecto que puede deberse a alguna alteración del tallo hipotálamo- hipófisis.

Por otra parte, en cuanto a los niveles de TSH se encontraron valores por debajo de lo normal, mientras que para T4L aunque estuvieron dentro del rango normal el valor fue bajo; lo que es un indicador de la presencia de algún factor que genera este cambio para el perclorato de amonio.

Para el caso del Grupo Dosis Duplicada, se observó, en cuanto al peso; que hubo una tendencia a estabilizarse después de la mitad del tratamiento. En cuanto a los niveles de TSH tuvieron tendencia a disminuirse frente al rango normal y la T4L libre salió de los límites normales con tendencia al aumento, lo que determinó una sobreproducción de la hormona y permitió establecer una relación con el comportamiento, ya que era un grupo de hiperactividad marcada.

De la misma forma, en el Grupo de Dosis Quintuplicada, el peso de los ratones; tendió a estanda- rizarse luego de la mitad del tratamiento, lo que corrobora que el peso no es un factor decisivo en el comportamiento de los niveles hormonales. Los niveles de TSH se encontraron disminuidos pero dentro del rango normal, mientras que T4L libre estuvo aumentada en todo el grupo.

En el último grupo, es decir dosis Decaplicada, el peso; se estandarizo y los valores de TSH tendieron a estar en su valor dentro del rango normal, mientras que T4L también subió a niveles máximos, es decir una sobreproducción hormonal, debido a la dosis alta de perclorato de amonio.

Es evidente que el uso de esta sustancia incide en la sobreproducción de T4L, porque a medida que la dosis va aumentando los niveles son muy altos sugiriendo un hipertiroidismo. Sin embargo, es importante revisar el efecto del perclorato sobre TSH, la cual muestra una tendencia marcada a permanecer dentro del valor normal.

Es necesario hacer una revisión del receptor para TSH en la glándula tiroides donde al unirse con su ligando origina el estimulo productor de T4, induciendo la producción de tiroglobulina, peroxidasa tiroidea, NIS y tiroxina. Este sistema de retroalimentación negativa intenta mantener constantes los niveles de hormonas tiroideas en sangre, aspecto que seguramente es alterado por el perclorato y las diferentes dosis, que inciden sobre el receptor generando un descontrol en la producción de hormonas tiroideas para este caso T4L.

El desarrollo de este trabajo permitió de una manera experimental analizar posibles alteraciones tiroideas en ratones después de exponerlos a perclorato de amonio, por medio de cuantificación de TSH y T4 Libre en sangre, igualmente se pudieron determinar los cambios en el estado comportamental de los ratones expuestos a distintas concentraciones de percloratos. 


\section{Agradecimientos}

A todos los estudiantes del programa de Medicina de la Universidad de Boyacá que pertenecen al Semillero de Investigación IMAN (Investigación en Modelos Animales).

\section{Referencias}

1. Eva D. McLanahan, M. E. Competitive Inhibition of Thyroidal Uptake of Dietary Iodide by Perchlorate Does Not Describe Perturbations in Rat Serum Total T4 and TSH. Environmental Health Perspectives: 2009:731-738.

2. Lewis E. Braverman, E. N. Effects of Six Months of Daily Low-Dose Perchlorate Exposure on Thyroid Function in Healthy Volunteers. The Journal of Clinical Endocrinology \& Metabolism. 2006:2721-2724.

3. DYKE, J. V. Perclorato y Yodo en muestras biológicas. Universidad de Texas. 2008; 13( 63): 73-77.

4. Lyn Patrick, N. Thyroid Disruption: Mechanisms and Clinical Implications in Human Health. Alternative Medicine Review. 2009:326-346.

5. Tarone, R. E. Epidemiología de la exposición al perclorato ambiental y función tiroidea: Una revisión completa. JOEM
American College of occupational and Enviromental Medicine. 2010:653-660.

6. Lyn Patrick, N. Iodine: Deficiency and Therapeutic Considerations. Alternative Medicine Review: 2008:116-127.

7. Amitai Y, W. G. Gestational exposure to high perchlorate. Thyroid. 2007:843-850.

8. Blount BC, P. J. Urinary perchlorate and thyroid hormone levels in adolescent and adult men. Environ Health Perspect. 2006:1865-1871.

9. Gibbs JP, A. R. Evaluation of a population with occupational exposure to airborne ammonium perchlorate for possible acute or chronic effects on thyroid function. J Occup Environ Med. 1998:1072-1082.

10. Greer MA, G. Health effects assessment for environmental perchlorate contamination: the dose response for inhibition of thyroidal radioiodine uptake in humans. Environ Health Perspect; 2002:927-937.

11. Pretell, E. A. et al. Consenso sobre los desórdenes por deficiencia de Yodo en Latinoamérica. Criterios de evaluación y monitoreo para su erradicación sostenida. Revista cubana de endocrinología. 1999;10 (2): 146-156.

12. Trumbo, P. R. Perchlorate consumption, iodine status, and thyroid function. Nutrition Reviews. 2009:62-66. 